\title{
The Relativistic Nature of Entropy Changes
}

\author{
Claudio Zamitti Mammana ${ }^{1}$
}

Received: 26 February 2021 / Accepted: 27 May 2021 / Published online: 21 June 2021

(c) The Author(s) 2021

\begin{abstract}
This paper presents a method to obtain the variations of the entropies of the phases of a chemical substance in its vapor state, which allows deriving, from thermodynamics, the axioms of a quantum theory that conforms to special relativity.
\end{abstract}

Keywords Phase transition kinetics $\cdot$ Entropy of vapor $\cdot$ Ladder operators $\cdot$ Time asymmetry

\section{Introduction}

Let us start by recalling some fundamental discoveries that occurred before the Copenhagen Interpretation of quantum mechanics:

1. In 1738, Daniel Bernoulli showed that a system of particles endowed with momentum degrees of freedom, enclosed inside a container of fixed volume $V$, exerts a pressure $p$ on its internal surface. Its energy $E$ is then,

$$
E=\frac{3}{2} p V
$$

2. Around 1850, Clausius revealed the existence of a hitherto unknown magnitude conjugated to the temperature, the entropy $S$, which allowed him to demonstrate the asymmetry of time and contributed with the mathematical conditions for the eventual formulation of the fundamental identity of thermodynamics,

$T d S=d E+p d V$.

3. During the years 1856-1860, Kirchhoff established that the chromatic distribution of electromagnetic radiation of the black body depends exclusively on its temperature. He also defined the hollow (Hohlraum), a hypothetic adiabatic container conceived to retain a finite amount of radiation.

Claudio Zamitti Mammana

mammana@if.usp.br

1 Institute of Physics, University of Sao Paulo, Sao Paulo, Brazil
4. In 1862, Maxwell revealed that electromagnetic radiation is endowed with momentum, exerting pressure on any surface to which it is exposed to.

5. In 1876, based on Maxwell's revelation, A. Bartoli redefined radiation as a Bernoulli's discrete fluid [1].

6. In 1884, adopting Bartoli's redefinition of heat, and using the laws of thermodynamics, Boltzmann derived Stefan's radiation law from the equation [2],

$E=3 p V$.

7. In 1900, Planck obtained the chromatic distribution of energy of radiation, introducing his constant $h$ into thermodynamics.

8. In 1907, Einstein derived a quantum-thermodynamic expression for the specific heat of solids, explaining their departure from the Dulong-Petit law at low temperatures.

9. In 1911, Sommerfeld interpreted the identity

$\Delta p \Delta q=h v$

as a taut constraint and introduced the dimensionless number, known as degree of gas degeneration [3],

$\rho=\frac{N}{V} \frac{h^{3}}{(\sqrt{2 \pi m k T})^{3}}$

10. In 1911-1912 Sackur and independently, Tetrode, using equation, deduced that the entropy of a monoatomic gas, revealing that it is a function of a single variable $\rho$.

11. In his derivation of Planck's radiation formula in 1917, assuming that heat has a momentum degree of freedom, Einstein concluded for the existence of three dif- 
ferent processes of quantum exchange, namely, absorption, spontaneous emission (which recalls a radioactive reaction), and stimulated emission.

From Einstein's theory of radiation, one can conclude that these processes are both mutually exclusive (no two of them happen simultaneously) and individually stochastic (the waiting time between two consecutive events is a random variable). Restating Einstein's assumptions in terms of the theory of stochastic processes, I arrived at the following theorem [4]:

$\$ 1$ The time evolution of the probabilities of the occupation numbers of the quantum states in the gases of Bose and Fermi particles can be described by a Markov birth-anddeath process whose laws of change derive from the conditional probabilities that characterize the ladder operators of their particles.

Note that fluctuation in equilibrium and time asymmetry are mere corollaries of theorem $\S 1$.

Being or Becoming?. By connecting absorption to emission into a single state transition matrix, Matrix Mechanics assumes that these processes are concomitant. Therefore, if Einstein's theory is correct, Matrix Mechanics holds only for systems in thermodynamic equilibrium.

It is remarkable that thermodynamics, instrumental for the empirical characterization of the matter-radiation interaction (the phenomenon which gives rise to quantum mechanics), has been so neglected after the development of matrix and wave mechanics.

The description of the thermodynamic equilibrium formation of the black body radiation in a hollow, given by Max Planck in his Scientific Biography, provides an enlightening interpretation of the results of the theorem $\S 1$, and justifies Einstein's 1917 approach:

"I assumed the cavity to be filled with simple linear oscillators or resonators, subject to small damping forces and having different periods; and I expected the exchange of energy caused by the reciprocal radiation of the oscillators to result, in time, in a stationary state of the normal energy distribution corresponding to Kirchhoff's Law."

The current explanation for the collective behavior of the gases of Bose and Fermi particles derives from the assumption that assigns either to their wave functions or to their particles the following ontological characters:

- While the wave functions of Bose particles are symmetric, Fermi's are anti-symmetric.
- The particles in a Bose gas are indistinguishable while those in a gas of fermions are compelled to occupy the quantum states according to the Pauli Exclusion Principle.

Nevertheless, besides theorem $\S 1$, other explanations for the indistinguishability of identical particles can be found:

\$ 2 During a chemical reaction, the degrees of freedom of the molecules of the reactants are annihilated, losing their classical identities, while new degrees of freedom, with unpredictable values, are created in its products.

In fact, according to quantum mechanics, the internal state of an atom or of a molecule changes only when they exchange one quantum of action with radiation. When two molecules $A$ and $B$ react to form the molecule $A B$, the degrees of freedom of both, $A$ and $B$, are destroyed, while those of the molecule $A B$ acquire new values, which depend on the way $A$ and $B$ interact with radiation.

Besides, classical predictability becomes impossible due to the indistinguishability of particles, which, according to theorem $\S 1$, results from the stochastic processes that lead the system to equilibrium.

Let us look at this phenomenon from a different standpoint. To describe the state of motion of a classical system composed of $f$ degrees of freedom, it is necessary to give $2 f$ real values of its dynamic variables. In quantum mechanics, differently, a system is described by only half that number. The reduction in the amount of information required to characterize the quantum system has been hitherto attributed to the restrictions imposed by the uncertainty principle [5].

The mechanics of Lagrange provides a different interpretation: It assigns the reduction of the number of degrees of freedom to the existence of constraints. As stated in $\$ 2$, a chemical reaction destroys the degrees of freedom of a molecule. We can therefore assume that during the reaction, the motion of the particles is subject to a constraint, namely, the Sommerfeld taut constraint (4).

Note that the explanations given above have nothing to do with the Copenhagen interpretation of quantum mechanics, which assigns to the so-called uncertainty principle the consequences of the constraint $\Delta p_{\ell} \Delta q_{\ell} \geq h$.

It will be shown in Section 3 that, by assuming that the laws of change in theorem $\S 1$ derive from the ladder operators, we are admitting their precedence over Pauli's exclusion and the indistinguishability principles, which, are not ontological characters of the particles but, instead, mere conditions of thermodynamic equilibrium. Hence, displacing a system of bosons from equilibrium allows distinguishing some of them from others; removing a gas of fermions from its stationary state might force more than one fermion to occupy the same quantum state. 


\section{The Perfect Vapor}

Two hypothetical mechanical models were used to simulate the behavior of matter under particular conditions:

1. The Bernoulli discrete fluid, was used in

- the foundations of the kinetic theory of gases, mainly by Maxwell and Boltzmann;

- by Boltzmann in his derivation of Stefan's law of radiation;

- by E. Leib as an abstract model (the Perfect Vapor) for a substance in the vapor state [6], reformulated in the forthcoming Sections;

2. The system of resonators was used by

- Boltzmann (1866), in his theoretical derivation of the Dulong-Petit empirical law (1819);

- Planck (1900) in the derivation of the black body radiation law;

- Einstein (1907) in the quantum derivation of the specific-heat of solids, later improved by Debye (1912).

- in the derivation of the consequences of the ladder operators on the population of quantum states.

Equation of State. Hereafter we will resume the BartoliBoltzmann approach to the discrete fluid by treating it as a thermodynamic system, which allows addressing the hitherto open problem in Kinetic Theory, namely to provide the methods:

- to predict the thermodynamic properties of a chemical substance in its vapor state;

- to describe the elementary laws that rule the motion of its particles during a chemical reaction.

This stratagem starts with subjecting the Equation (1) to the laws of thermodynamics (2). For generality, we will rewrite that equation in the form $E=\gamma p V$, where $\gamma$ is a rational number.

To obtain the functional relations involving the thermodynamic variables of these two families of discrete fluids under the constraints imposed by the second law, we subject equation $E=\gamma p V$ to the Maxwell relation ${ }^{1}$,

\footnotetext{
1 The conditions imposed on the gas here are essentially the same obtained by Clapeyron in 1834 to derive equation $\frac{d p}{d T}=\frac{\Delta H}{T \Delta V}$, known as the first physicochemical application of the second law of thermodynamics [7].
}

$\left(\frac{\partial E}{\partial V}\right)_{T}=T\left(\frac{\partial p}{\partial T}\right)_{V}-p$

Substituting $E$ by $\gamma p V$ in (6), we obtain,

$T\left(\frac{\partial p}{\partial T}\right)_{V}-\frac{1}{\gamma} V\left(\frac{\partial p}{\partial V}\right)_{T}=\frac{1}{\gamma+1} p$,

Being equation (7) a linear PDE of the first order, its solution depends on an arbitrary function $\varphi$ with a determined $\operatorname{argument}^{2}$,

$z=\varphi\left(T^{\gamma} V\right)$

where $z=p V / R T$. Since in physics the argument of an indeterminate function must be dimensionless, we conclude that the argument $\theta$ of the equation of state (EOS) $z=\varphi(\theta)$ of the Perfect Vapor, can be given by the formula:

$\theta=\left(\frac{h}{\sqrt{2 \pi m k T}} \sqrt[3]{\frac{N}{V}}\right)$

where the dimensionless number $\theta$ is the cubic root of the degree of gas degeneration (5).

The equation of state (8) for $\gamma=3$, which represents electromagnetic radiation, acquires the well-known form,

$z=\varphi\left(\frac{h v}{k T}\right)$

Equations (1) and (3) lead to the EOs (8), expressed by functions of a single argument. Hence, according to the phase rule, the substances they define are two-phase systems. The former will be here adopted as the definition of the Perfect Vapor, and the latter is the subject of the Bose-Einstein condensation theory.

Asymptotic Behavior. It is known that for a chemical substance $\sigma$, we have,:

$\lim _{\theta \rightarrow 0} \varphi_{\sigma}(\theta)=1$.

It is worthwhile to express $z=\varphi_{\sigma}(\theta)$ in terms of the power series expansion,

$z=1-\theta\left(a_{1}+a_{2} \theta+a_{3} \theta^{2}+\ldots\right)=1-f_{\sigma}(\theta)$,

where the $a_{i}$ 's are constants, and $f_{\sigma}(\theta)$ is a function that characterizes the indeterminate substance $\sigma$ of the single argument $\theta$. Expressed in terms of thermodynamic variables, it acquires the form,

\footnotetext{
2 The derivation of the equation of state (8) of the Perfect Vapor is a translation to the native variables thermodynamics of the abstract model proposed by Leib [6].
} 
$p V+R T f_{\sigma}(\theta)=R T, \quad f_{\sigma}(0)=0$,

For illustration pusporses the equation of state (11 is confronted against the steam $p V T$ data in Appendix 1.

The Entropy of the Perfect Vapor. Let us subject the equation of state (11), to the Maxwell relation,

$\left(\frac{\partial S}{\partial V}\right)_{T}=\left(\frac{\partial p}{\partial T}\right)_{V}$

which leads to a different PDE, whose solution, $S=S_{\mathscr{G}}+S_{\mathfrak{L}}$, is given by the following expressions,

$S_{\mathscr{G}}=-3 R \ln \theta$,

$S_{\mathfrak{Q}}=-\frac{3}{2} R f_{\sigma}(\theta)+3 R \int \frac{f_{\sigma}(\theta)}{\theta} d \theta$,

In expression (13), one can recognize the Sackur-Tetrode entropy of the ideal monoatomic gas. The total entropy of the perfect vapor arises as the sum of two terms, which, according to Boltzmann's principle, are statistically independent.

After the entropies (10) and (11), the Perfect Vapor can be treated as an extension of the abstract notion of the Perfect Gas (10) to describes a two-phase system, in particular, the vapor.

So far, we have been able to determine the entropy of the liquid phase (14) from the equation state (1) by purely thermodynamic methods. However, to determine the unknown function $f(\theta)$, we must have recourse to special relativity, as shown in Section 3.

Modeling the Liquid Phase. To describe the phases of the perfect vapor, we will have recourse to the notions of radicle and cluster, defined as follows:

Radicle. Let's assume that during vaporization, the perfect vapor molecules do not dissociate themselves into smaller molecules. Instead, during these phase transition processes, they behave as indivisible units, as it happens with the atoms in the molecules in chemical reactions. Molecules with this chemical behavior were named radicles by Berzelius [8].

Cluster. A cluster is a molecule composed of a random number $\mathbf{r}_{\ell}(t)$ of radicles.

The liquid phase will be here treated as a many-body quantum system composed of a random number of clusters. This model of the liquid phase of the perfect vapor allows derive its "laws of motion" described, not in terms of the trajectories of its particles, expressed by their positions and velocities as functions of time, but in terms of the time evolution of the Probability Distribution Function (PDF) of the occupancy random numbers of the quantum states of a many-body system [4].

\section{Matter-radiation Equilibrium}

In this section, we will resume Kirchhoff's adiabatic hollow, placing inside it a portion of liquid, to analyze the vaporization process and the formation of its equilibrium, for this case is entirely analogous to a mixture of liquid and vapor enclosed in a cylinder with a moving piston ${ }^{3}$. Since both equations, (1) and (3), define thermodynamic systems, we are allowed to study their equilibrium according to the following theorem:

$\$ \mathbf{3}$ “(...) in any reversible process a system, or any part of a system, undergoes an increase in entropy as it absorbs heat from the medium, resulting in an equal decrease in the entropy of the medium, and that the increase in entropy is equal to the heat so absorbed divided by the absolute temperature, $d S=\frac{\delta q_{\text {rev }}}{T}$." [7]

The following corollary is derived from Theorem $\S 3$ : Ehrenfest Adiabatic hypothesis. During an adiabatic process, the mechanical action is conserved.

Therefore, in the thermodynamic equilibrium, the average variation of their phase-space volumes must be equal:

$\frac{k T c}{\lambda}=\sqrt{2 \pi m k T} \sqrt[3]{\frac{V}{N}}$.

By adopting the approximation $\langle\lambda\rangle \propto\left\langle\sqrt[3]{\frac{V}{N}}\right\rangle$ for the wave length of radiation, we obtain, from (15), the proportionality relation $k T \propto m c^{2}$, which reveals that the motion of molecules under the action of thermal radiation is relativistic.

In the first law of thermodynamics, heat is treated as a scalar amount of energy, which is converted into work, through a undetermined microscopic conversion processes. Although the entropy of the liquid phase 14 can be obtained from the equation of state, by an exclusively thermodynamic method, it depends on the indeterminate function $f(\theta)$. The determination of its functional form, as shown in the sequence, requires having recourse to special relativity.

To ensure epistemological consistency between the definition of the discrete fluid and its thermodynamic consequence (9), we represent the energy $E$ according to the expression,

$E^{2}=m^{2} c^{4}+c^{2} p^{2}=\left(m c^{2}+\imath c p\right)\left(m c^{2}-\imath c p\right)$.

$\overline{3 \text { p. } 111 \text { [7] }}$ and $\S \S 51,52$ [9]. 
It is possible to give a thermodynamic meaning to equation (16) by having recourse to quantum mechanics. In this undertaking, however, we must move a step backward towards the Old Quantum Theory, adopting the hypotheses below:

1. Sommerfeld's constraint. That the relation $\Delta p \Delta q \geq h$, currently interpreted according to Heisenberg's uncertainty principle will be here reinterpreted according to the Sommerfeld taut constraint (4).

2. Einstein 1917 paper. In the dynamics of the thermodynamic equilibrium formation, here proposed, I assume, with Einstein's Quantum Theory of Radiation, the following hypotheses:

1. that the three processes, namely, absorption and spontaneous and stimulated emissions, are mutually exclusive random processes and their occurrences are stochastically independent;

2. that thermodynamic equilibrium is the result of the persistent action of these elementary processes.

3. Einstein-Bohr relation. That the energies exchanged during the absorption and emission of quanta processes are expressed by the equations,

$\Delta E=\Delta m c^{2}+\imath c \Delta p$

$\Delta E^{*}=\Delta m c^{2}-\imath c \Delta p$,

which supersede the current Einstein-Bohr relation.

4. Stochastic randomization. That the interaction of matter with radiation predicted by Einstein occurs according to theorem $\S 1$, which characterizes a Markovian stochastic process:

- the continuity hypothesis: The smaller the time interval considered, the smaller the number of clusters changing their states.

- the independence hypothesis: The removal of any cluster (together with the radicles it contains) from the vapor will not modify the flow processes that take place in the remaining clusters. In other words, the flow of radicles in a given cluster is independent of the flow that occurs in any other cluster.

Complex Volume of the Phase Space. The variation $\Delta \Omega$, of the perfect vapor phase-space volume during the time interval $\Delta t$, when the gas and radiation exchange one quantum of action, is given by either of the equations,

$\Delta \Omega=\Delta E \Delta t=\Delta m c^{2} \Delta t+\imath c \Delta p \Delta t$,
Rewritten in their dimensionless terms, we have,

$\frac{\Delta \alpha}{h}=\frac{\Delta p \cdot \Delta z}{h}+l \frac{\Delta p \times \Delta z}{h}$,

$\frac{\Delta \alpha^{*}}{h}=\frac{\Delta p \cdot \Delta z}{h}-\imath \frac{\Delta p \times \Delta z}{h}$,

where from we obtain,

$\left(\Delta \alpha-\Delta \alpha^{*}\right)=2 \imath(\Delta p \times \Delta z)$.

Equations (19) and (20) supersede the equations of the harmonic oscillator (the Planck resonator) in the current derivation of the mathematical expressions of the ladder operators in quantum mechanics ( $\$ 41$, Ch. III [5]), which reveals that these operators determine the laws that rule the absorption and emission processes of quanta exchange.

Equations (19) and (20) introduce the imaginary unit $l$ coupled to Planck's constant $h$ in quantum mechanics. We can then interpret this couple as an imaginary constant endowed with the faculty to transform a difference operator to which it applies, into a differential operator.

\section{Matter-radiation Interaction}

For the particles moving in the classical subspace of the phase space, $\Delta \alpha_{j}>h$, the Boltzmann entropy is given by

$\Delta \mathrm{S}_{j}=\ln \left(\frac{\left|\Delta p_{j} \cdot \Delta q_{j}\right|}{h}\right)$.

Denoting the variations of the actions (19) and (20) by the conventional symbols $\Delta \alpha\left(\mathrm{a}^{\dagger}\right)$ and $\Delta \alpha(\mathrm{a})$, respectively, we can write the variation of Boltzmann's entropy in terms of the complex logarithmic function (Ln), when the particles of the gas are under the action of radiation, i.e., when $\Delta \alpha_{j}=h$, a cluster in the liquid phase absorbs or emits one quantum,

$\Delta \mathrm{S}_{k}=\left\{\begin{array}{l}\operatorname{Ln}\left[\Delta \alpha_{k}\left(\mathrm{a}^{\dagger}\right)\right]=\operatorname{Ln}\left[\left(\Delta p_{k} \cdot \Delta q_{k}\right)-\imath\left(\Delta p_{k} \times \Delta q_{k}\right)\right], \\ \operatorname{Ln}\left[\Delta \alpha_{k}(\mathrm{a})\right]=\operatorname{Ln}\left[\left(\Delta p_{k} \cdot \Delta q_{k}\right)+\imath\left(\Delta p_{k} \times \Delta q_{k}\right)\right] .\end{array}\right.$

The Entropy of the Liquid Phase. The change of the entropy caused by the "stochastic equilibrium" of the two opposite processes of absorption and emission, is given by the amount,

$\operatorname{Ln}\left(\mathrm{a}_{k}^{\dagger}\right)-\operatorname{Ln}\left(\mathrm{a}_{j}\right)=\operatorname{Ln}\left[\frac{\left(p_{k} \cdot q_{k}\right)-\imath\left(p_{k} \times q_{k}\right)}{\left(p_{j} \cdot q_{j}\right)+\imath\left(p_{j} \times q_{j}\right)}\right]$.

Denoting the quotient, 
$\vartheta=\frac{p \times q}{p \cdot q}$

we can rewrite equation ( 22 in the dimensionless form,

$\left(\frac{a_{k}^{\dagger}}{a_{j}}\right)=\frac{1-\imath \vartheta_{k}}{1+\imath \vartheta_{j}}$.

Both the quantities $\vartheta_{k}$ and $\vartheta_{j}$ are described by the same PDF. Denoting by $g(\theta)=\vartheta_{k}=\vartheta_{j}$, the function of the single argument $\theta$ and substituting in (24), we have,

$\frac{S_{\mathfrak{Q}}}{k}=\imath \operatorname{Ln}\left(\frac{\left\langle a^{\dagger}\right\rangle}{\langle a\rangle}\right)=2 \arctan (g(\theta))$,

which, as expected, is a scalar magnitude.

Recalling that the partition function of the black body radiation is given by,

$\frac{1}{2} \operatorname{csch}\left(\frac{h v}{2 k T}\right)$

From identity (15), we obtain the entropy of the liquid phase in equilibrium with the black body,

$\frac{S_{\mathfrak{Q}}}{k}=\left\{\left(j+\frac{1}{2}\right) \pi-\arctan \left[2 \sinh \left(\frac{\theta}{2}\right)\right]\right\}$,

where the integers $j=1,2, \cdots$ might represent the contribution of some yet unknown phenomenon.

From equation (27) derives the EOS (11) of the perfect vapor ${ }^{4}$ where,

$f(\theta)=\frac{\theta}{2} \operatorname{sech}\left(\frac{\theta}{2}\right)$.

It is remarkable that equation (24) describes a conformal mapping, thus justifying Prévost's theory of heat exchange.

\section{Space-time X Timeless Space}

The four-dimensional interval $d S$ has two distinct representations: in the space-time and in the timeless Minkowskian space. While in the Minkowski space, it has the following timeless representation,

$d s^{2}=x_{1}^{2}+x_{2}^{2}+x_{3}^{2}+x_{4}^{2}$,

in the space-time its representation is,

\footnotetext{
${ }^{4}$ In (28)is an odd function, as expected from the clear half-turn symmetry observed in the vapor region $\left(\zeta<\theta^{*}\right)$, Fig. 2, although around a yet indeterminate center.
}

$d s^{2}=\underbrace{c^{2} t^{2}-\overbrace{\left(x_{1}^{2}+x_{2}^{2}+x_{3}^{2}\right)}^{\text {Euclidean }}}_{\text {space-time }}$,

which describes the relativistic restriction (constraint $|v| \leq c)$, imposed on the motion of Newtonian particles.

The radical difference between the structures of the gaseous and liquid phases suggests that the equations (29) and (30) describe distinct realities and, therefore, are not equivalent. While the former applies to the liquid phase, characterized by molecules, whose structure is stationary and involves a non-observable variable, the spin, which is defined in the timeless Minkowski domain, the latter applies to the gaseous phase, which is a system of classical particles evolving in the space-time, where they can, in principle, be observed.

Let us decompose Dirac's momentum matrix [10] into its real and imaginary parts,

$\left(\begin{array}{cccc}m c & 0 & \hat{p}_{z} & \hat{p}_{x} \\ 0 & m c & \hat{p}_{x} & -\hat{p}_{z} \\ \hat{p}_{z} & \hat{p}_{x} & -m c & 0 \\ \hat{p}_{x} & -\hat{p}_{z} & 0 & -m c\end{array}\right)+\imath \hat{p}_{y}\left(\begin{array}{cccc}0 & 0 & 0 & -1 \\ 0 & 0 & 1 & 0 \\ 0 & -1 & 0 & 0 \\ 1 & 0 & 0 & 0\end{array}\right)$

Note that the momentum operators $\hat{p}_{x}$ and $\hat{p}_{y}$ are conjugate in the $x y$ complex plane. Since the momentum $\hat{p}_{y}$ is proportional to $h$, it is very close to the real line in the complex plane, no matter how far its remaining coordinates are found. We say that, in the formation of such a system, the particles become entangled.

Radiation and Schrödinger Equation. The evidence provided by $\S$, leads us to inquire on the following question:

How the independent degrees of freedom of a radicle in the gaseous phase of the perfect vapor become, as the consequence of a chemical reaction, conjugate inside a cluster?

The variation $\Delta E$ of the energy is given by the finite work performed by the 'force' $\dot{p}$,

$\Delta E=\frac{\Delta p_{\ell}}{\Delta \tau} \Delta q_{\ell}=h v$.

If this algebraic manipulation is justified, we are allowed to assume that $\Delta E$ corresponds to a non-conservative process.

The connection between the pairs of degrees of freedom $\left\{p_{\ell}, q_{\ell}\right\}$ or between $\{E, \tau\}$, can be justified when we realize that Planck's constant cannot be interpreted as a mere scalar magnitude. Due to its enigmatic nature, the mathematical interconnection of these pairs of quantities were given in the formulation of wave mechanics by an unconventional 
treatment of the mathematical terms involved, as follows. Rewriting equations $\Delta E \Delta t=h$ and $\Delta p \Delta q=h$ in the forms,

$\Delta E=\frac{h}{\Delta t}$, and $\Delta p=\frac{h}{\Delta q}$,

the difference operators $\Delta$ become interpreted, in quantum mechanics, as the discrete version of the corresponding differential operators of energy and momentum, respectively,

$\lim _{\Delta t \rightarrow 0} \frac{h}{\Delta t} \rightarrow \hat{E}=\frac{h}{2 \pi l} \frac{\partial}{\partial t}$,

$\lim _{\Delta x \rightarrow 0} \frac{h}{\Delta x} \rightarrow \hat{p}_{x}=\frac{h}{2 \pi l} \frac{\partial}{\partial x}$.

The correspondences (33) and the algebraic properties of operators adopted in quantum mechanics establish the rules necessary to formulate the operators that characterize atoms in atomic theory and molecules in chemistry, as many-body quantum systems. We can then interpret these operators as conjugators, i.e., entities that merge the degrees of freedom of the particles of a system, originally independent, into a single conjugated unit, imposed on them by the action of radiation, thus destroying half of their original degrees of freedom.

\section{Frequency Match Principle}

Mathematical Representation of Spectra. In statistical physics, it is customary to describe the discrete frequency spectrum of a system by the generating function [11] (GF) defined in Operational Calculus. Considering that the equilibrium formation between radiation and the chemical substance is a stochastic process, we adopt the random integer occupancy numbers $\mathbf{r}_{\ell}$ of the quantum states as independent coordinates; as the dependent variable, we adopt the corresponding probability $\operatorname{Prob}\left(\mathbf{r}_{\ell}=k\right)$ of finding $k$ particles in the state $j$.

The GF of the PDF of the equilibrium occupancy random integer variable $\overline{\mathbf{r}}_{\ell}$ is well-known from the description of radiation in terms of the quantum harmonic oscillator given by equation (26).

Metastable States. The description of a thermodynamic system requires knowledge of the spectrum of radiation in its environment. Recall that no chemical substance is susceptible to frequencies different from those characterized by its molecular structure.

When the black-body radiation interacts with a chemical substance, the latter will eventually attain its state of stable equilibrium. An arbitrary radiation source might either cause no change in the substance or lead it to some metastable equilibrium state.
The above considerations are relevant if the chemical substance can be found in different metastable states, as it happens with clouds in the atmosphere. It is well known that dry air is transparent to most of the frequencies found in solar radiation. However, it is sensitive to the radiation emitted by the earth's surface, which functions as a geographically distributed set of frequency transducers. Its metastable states explain the variety of cloud formations observed.

The Equilibrium Condition. Considering that absorption and emission processes are mutually independent, equilibrium only occurs when the following equation is satisfied ${ }^{5}$,

$\frac{\langle\Delta \alpha\rangle}{\left\langle\Delta \alpha^{*}\right\rangle} \approx 1$.

Hence, a many-body system attains its equilibrium state only when the number of absorptions is statistically equal to the number of emissions, i.e., when the average actions accumulated during these processes compensate each other.

During the absorption or the emission processes, photons are neither annihilated nor created but merely exchanged between matter and radiation.

Random Variables in the Liquid Phase. We can depict an image of the liquid phase of the perfect vapor as a random number $\mathbf{L}$ of clusters, where a given cluster $\ell$ is a "molecule" composed of a random number $\mathbf{r}_{\ell}$ of radicles of the perfect vapor, characterized by the quantum number $\ell$. Taking into account that these random variables are stochastically independent, the number of radicles in the liquid phase, in each moment, can therefore be given by the random number $\mathbf{S}_{\mathbf{L}}$, expressed by the sum,

$\mathbf{S}_{\mathbf{L}}=\mathbf{r}_{1}+\mathbf{r}_{2}+\cdots+\mathbf{r}_{\ell} \cdots+\mathbf{r}_{\mathbf{L}}$

Equation (35) characterizes a compound process. If we denote by

$\beta(s)=\beta_{1}(s) \cdot \beta_{2}(s) \cdots \beta_{\mathbf{L}}(s)$,

the convolution of the generating functions of the independent random variables $\mathbf{r}_{\ell}$, then the $\mathrm{GF}$ of the random variable, $\mathbf{S}_{\mathbf{L}}$, is given by $g(s)=g(\beta(s))[11]$.

Since this function is an exclusive function of $\theta$, we can replace $s$ by $\theta$, obtaining,

$g(s)=g(\beta(\theta))$.

\footnotetext{
5 The symbol " $\approx$ " is here used to denote the fluctuation of the values of the quantities involved
} 
The quantity $\vartheta$, defined in (23), can then be expressed in terms of a function $\vartheta(\theta)$ of $\theta$. Hence, the relation (25) can be written in the form,

$\arctan \left(\vartheta_{j}\right)=\arctan (\vartheta(\theta))$.

Migration of Radicles Between Phases. During the absorption or emission of one quantum, there is a corresponding exchange of radicles between the liquid and the gaseous phases. This process takes place according to the laws that rule the action of the creation-annihilation operators. Being independent and recurrent, they bring the perfect vapor to equilibrium after the relaxation time exhausts. The thermodynamic equilibrium is not a stationary state but an incessant fluctuation of the number of radicles around its characteristic value $^{6}$.

Hence, we cannot interpret these operators literally as something that causes a particle to come from nowhere or destroy it to nothing. Instead, they merely describe the exchanging of particles between the phases $\mathfrak{G}$ and $\mathfrak{L}$.

The elementary exchange of heat between a molecule and radiation allows concluding that the occupation of photons in quantum states of radiation corresponds reciprocally to the occupancy of particles of the gas in the liquid phase. Hence, the form of the partition function for particles in the cluster of the quantum number $\ell$ is the same for photons in the quantum state $\ell$,

$\beta(s) \Rightarrow \frac{1}{2} \operatorname{csch}\left(\frac{\theta}{2}\right)$.

Since we refer to the perfect vapor, we replace the argument $s$ of the function $\beta(s)$ by $\theta$. The expression of the GF of the random variable $\mathbf{S}_{\mathbf{L}}$ then becomes,

$g\left(\frac{1}{2} \operatorname{csch}\left(\frac{\theta}{2}\right)\right)$

In the particular case in which the perfect vapor is under the influence of black-body radiation, the function $\vartheta(\theta)$ is given by (37), which gives the generating function of the thermodynamic properties of the perfect vapor's liquid phase, whose entropy is given by (27).

\section{Conclusions}

In addition to presenting the equation of state of the perfect vapor in interaction with electromagnetic radiation and explaining the mechanisms of entropy formation during the

6 The time-dependent PDF of the occupation numbers is given in [4] vaporization-condensation processes, the present paper also suggests a reinterpretation of the following axiom of quantum mechanics, which turns that theory consistent with both thermodynamics and special relativity:

Axiom. Aux coordonnées $x_{j}$ et aux impulsions $p_{j}$ (j étant le numéro du degré de liberté) correspondent dans le système de la mécanique quantique les opérateurs $X_{j}$ et $P_{j}$ satisfaisant aux relations de commutation

$X_{j} P_{k}-P_{k} X_{j}=\imath \hbar \delta_{j k} \mathbf{I}$

où $\hbar$ est la constante de Planck.[5]

It can be seen that the essence of this axiom arises naturally from the algebra that rules the relativistic nature of the matter-radiation interaction:

1. it introduces the unit of complex numbers, $l$, as a natural consequence of Minkowski's timeless representation of special relativity;

2. the commutation rule derives directly from complex algebra;

3. it reveals the relativistic nature of the spin, as an angular momentum degree of freedom;

4. it allows identify, in the imaginary part of the expressions of the ladder operators, the descriptors of the elementary processes of absorption and emission of quanta, as the causes of change of the volume of the phase space of the perfect vapor;

5. it introduces Planck's constant $h$ as an imaginary magnitude, which endows the physical variables it affects with the faculties of differential operators;

6. it gives precedence of the ladder operators over the indistinguishability and Pauli's exclusion principles, which arise as mere characters of thermodynamic equilibrium;

7. by assigning a different meaning to time, it replaces the partial differential time operator $\frac{\partial}{\partial t}$ in Schrödinger's equation by the Markov difference-differential equation, to describe the transient action of the ladder operators, thus predicting time asymmetry;

8. Eadem mutata resurgo. The incessant action of the ladder operators on the liquid phase clusters leads asymptotically to the same result given by Schrödinger's timeless amplitude equation, which, as an automorphism, accurately describes the equilibrium condition;

9. the stochastic nature of entropy formation processes cause the corresponding necessary collapses of wave functions, as the result of the persistent action of radiation on the quantum systems; 
Fig. $1 p V T$ data in the $\theta \times z$ plane

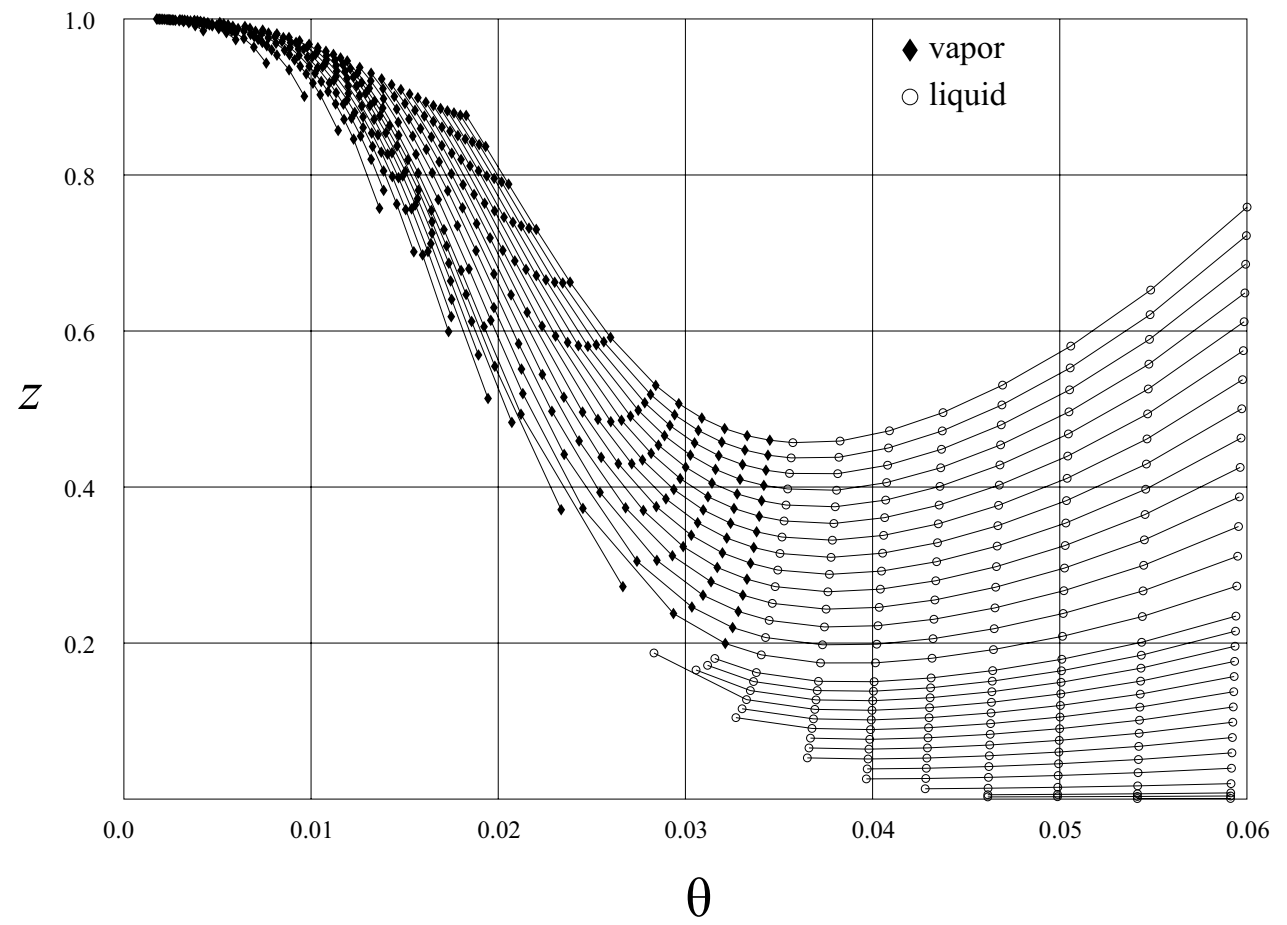

Furthermore, the present approach shows that the processes leading to the eigenvalues of wave functions are not concurrent, thus revealing that Schrödinger's cat paradox does not apply, nor does it acknowledges the debatable processes of observation and measurement as elements of explanation of quantum phenomena.

\section{The Perfect Vapor and Steam}

\section{Abstract}

This Appendix confronts the $p V T$ data of steam against the equation of state of the Perfect Vapor and discusses its departure from both the ideal gas law and the Perfect Vapor.

\section{Introduction}

While the indeterminacy of function $f(\theta)$ endows the perfect vapor with generality, its universality cannot be claimed until an extensive confrontation with the experiment is fulfilled, an endeavor that is beyond the scope of this paper.

Since $z$ and $\theta$ are both dimensionless quantities, the plot of the $p V T$ data of any substance in the $\theta \times z$ plane is meaningful for both a thermodynamic and a quantum reading. The closer these data are to a single curve ${ }^{7}$, the better the

\footnotetext{
7 The quotient obtained by dividing the area occupied by the $p V T$ data, by the total area determined by the selected intervals of $z$ and $\theta$, gives a rough measure of how close the substance is to the perfect vapor.
}

substance can be represented by Equation (11), where $f(\theta)$ represents the departure of the perfect vapor from the Clapeyron equation.

\section{The Departure of Steam from the Ideal Gas}

To form a rough idea of the functional form of $f(\theta)$, the graphic of $\theta \times z$ of the steam $p V T$ data $^{8}$ is exhibited in Fig. 1 .

The isobaric curves of steam form a family of isomorphic shapes, regularly displaced in the vertical direction, suggesting that the departure of the steam $p V T$ data from the ideal gas law $(p V=R T)$ is due to the composition of at least two phenomena: on one hand, the displacements explained by the perfect vapor, and on the other hand, the contribution of an independent phenomenon that causes the departure of the isobaric curves from the perfect vapor.

Considerations about the causes of the formation of the entropy of steam led me to assume that steam is described by the superposition of the Clapeyron-Clausius entropy variation during a phase transition to the perfect vapor behavior, supposedly described by the clustering of water molecules,

\footnotetext{
${ }^{8}$ Figures 1, 2 were introduced here for illustration purposes only. The data there exhibited were obtained from an old steam table I had at hand [12] when I programmed and used the algorithms to obtain the value of the parameter $p_{\ell}$, required to produce Fig. 2 . Since its determination was based on a low accuracy visual trial and error procedure, higher accuracy in the approximation (39) would be of little value.
} 
Fig. $2 p V T$ data in the $\theta \times \zeta$ plane

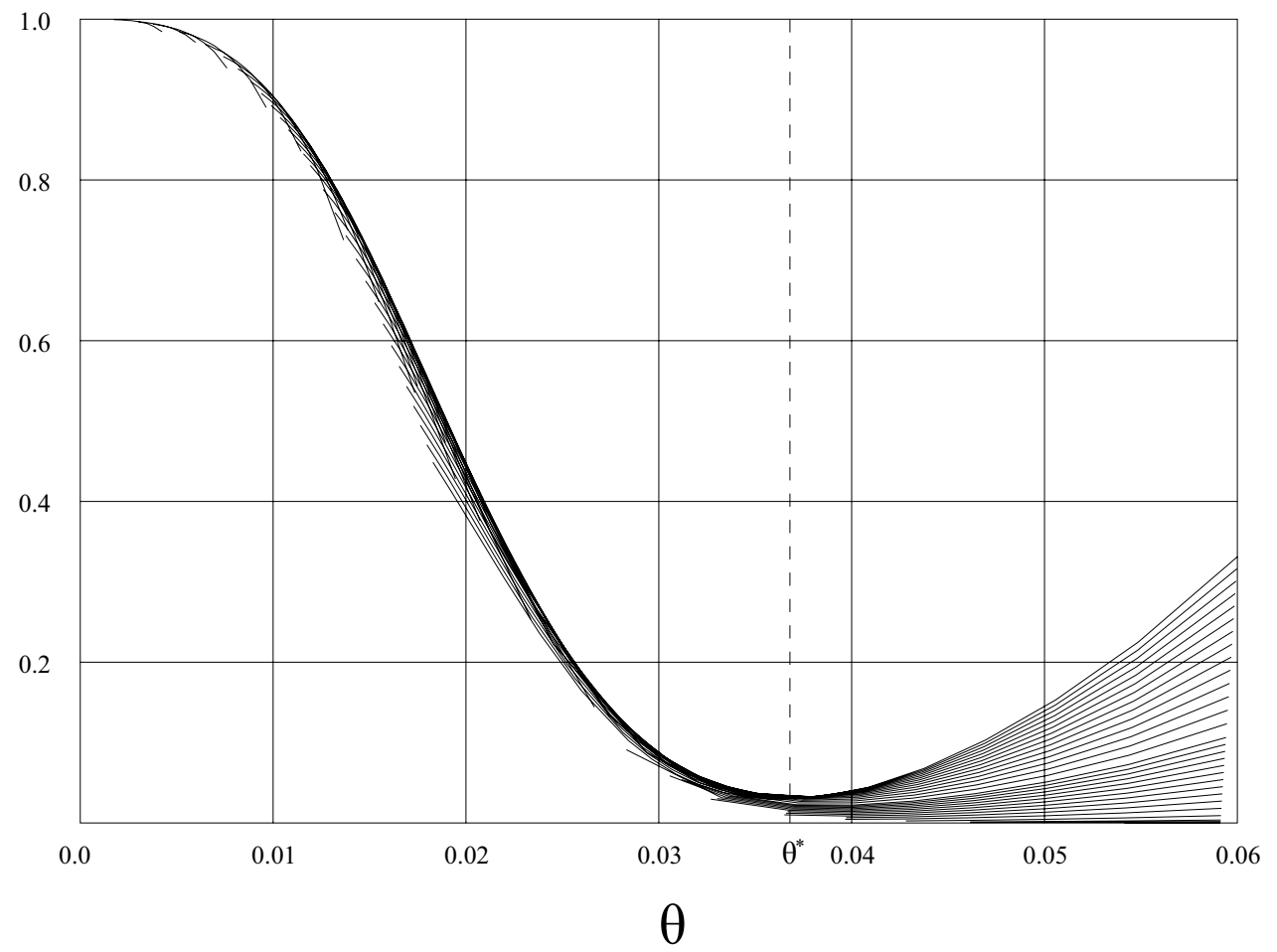

a hypothesis justified by its consequences. I, therefore, propose the following conjecture:

The departure of the isobaric curves from the perfect vapor is due to nucleation processes, and that nucleation and clustering are independent processes.

\section{The Departure of Steam from the Perfect Vapor}

To form an idea of the functional form of $f(\theta)$, it would be necessary to eliminate the influence of the unknown cause of the departure of the steam from the perfect vapor.

It is here conjectured that such departure is due to the nucleation phenomenon, by assuming that the vapor pressure $p$ of a substance is given by the equation,

$\frac{p}{p_{\ell}}=e^{-\frac{\Delta H}{R T}}$

where $\Delta H$ is the variation of the enthalpy of the system during a vaporization/condensation process, and $p_{\ell}$ is a constant pressure, characteristic of the liquid state.

For small values of $\Delta H / R T$, we can write $\exp (-\Delta H / R T)$ $\approx 1-\Delta H / R T$ to express the energy equation,

$\frac{p}{p_{\ell}} R T+\Delta H \approx R T=\frac{p}{p_{\ell}} R T+\Delta U+p \Delta V \approx R T$.

Since in a change of state $p \Delta V=V-V_{\ell}$ where $V$ is the volume of gas and $V_{\ell}$, that of the liquid, we can write $p \Delta V \approx p V$, thus obtaining, $p V+\frac{p}{p_{\ell}} R T+\Delta U \approx R T$,

Comparing the quantities $R T-p V$ obtained from Equations (11) and (39), we conclude that they represent different departures from the Clapeyron equation, due to distinct phenomena. By assuming that the behavior of the steam is the result of their combined action, we obtain its equation of state in the following form,

$z+f(\theta)+\frac{p}{p_{\ell}} \approx 1$.

Fig. 2 represents the steam data in the $\theta \times \zeta$ plane ${ }^{9}$, where,

$\zeta=z-p / p_{\ell}$.

By suppressing the influence of nucleation, the dispersion becomes largely attenuated, so that all isobaric curves in the vapor region become confined in a narrow belt, upperbounded by a single, sharp limiting curve. Denoting the area occupied by the belt of curves by $S_{b}$ and by $S_{r}$ the area of the rectangle defined by the opposing vertices $A=[0,1]$ and $B=\left[\zeta^{*}, \theta^{*}\right]$, we can say that the Perfect Vapor properly represents the thermodynamic properties of within the accuracy $\epsilon \approx S_{b} / S_{r}$.

\footnotetext{
9 The value of $p_{l} \approx 2341$ bar in equation (40) was obtained by a lowaccuracy visual trial and error method, that made the consideration $L / R T$ superfluous for a reliable estimation of $p_{\ell}$.
} 


\section{Remarkable Symmetries in Steam}

The rectangle determined by the vertices $A$ and $B$ delimit the vapor region ${ }^{10}$. It can be seen that around the mid-point of the diagonal $A B$ the curve exhibits a remarkable half-turn symmetry, which allows concluding that $B$ is symmetric to $[0,1]$, which is confirmed by the theoretical curve described by (28), which is an odd function, whose origin is in the midpoint of the diagonal $A B$ of the rectangle.

By rotating Fig. 1 of $180^{\circ}$, it is possible, by graphic translation, make the boundary curves coincide. Denoting by $A^{\prime}$ and $B^{\prime}$ the opposing vertices of the rotated curve, we have, $A^{\prime}=B$ and $B^{\prime}=A$, which allows obtain graphically the values of $\theta^{*}$ and $\zeta^{*}$.

It can be seen that, the greater the value of $\theta$ in the vapor region, the more degenerate is the gas and the closer it is to its liquid state. It is interesting to confront these symmetries against those that arise in the quasi-particles representation of collective phenomena.

Open Access This article is licensed under a Creative Commons Attribution 4.0 International License, which permits use, sharing, adaptation, distribution and reproduction in any medium or format, as long as you give appropriate credit to the original author(s) and the source, provide a link to the Creative Commons licence, and indicate if changes were made. The images or other third party material in this article are included in the article's Creative Commons licence, unless indicated otherwise in a credit line to the material. If material is not included in the article's Creative Commons licence and your intended use is not permitted by statutory regulation or exceeds the permitted use, you will need to obtain permission directly from the copyright holder. To view a copy of this licence, visit http://creativecommons.org/licenses/by/4.0/.

\section{References}

1. B. Carazza, H. Kragh, Afolfo Bartoli and the Problem of Radiation Heat. Ann. Sci. 46, 183-194 (1988)

2. J. Crepeau, Proceedings HT-2009, asme Summer Heat Conference, US (2009)

3. A. Sommerfeld, Thermodynamics and Statistical Mechanics (Press, Acad, 1956)

4. C. Z. Mammana, Phys Ess., 10(4), pp 608-14 (1997)

5. E. Chpolski, Physique Atomique, tome ii,mir, Moscow (1978)

6. E.F Leib, The Thermodynamic Properties of Vapors, Transactions of the A.S.M.E., 157-176 (1941)

7. K. Pitzer, L. Brewer, Thermodynamics, revision of G.N Lewis, M. Randall, Thermodynamics, McGraw-Hill (1961)

8. C. Singer, A Short History of Science to the Nineteenth Century, Oxford (1941)

9. M. Planck, The Theory of Heat Radiation, dover (1959)

10. A.S. Kompaneyets, Theoretical Physics, mir, Moscow (1961)

11. W. Feller, An Introduction to Probability Theory and its Applications, vol. i, John Wiley \& Sons, Inc. (1968)

12. R.W. Haywood, Sixth International Conference on the Properties of Steam - Release, J. of Eng. Power, Jan (1965)

Publisher's note Springer Nature remains neutral with regard to jurisdictional claims in published maps and institutional affiliations.

${ }^{10}$ It can be seen that the value $\theta^{*}$ at $B$ separates the vapor from the liquid phase of water. 